\title{
IBN BĀŶŶA (AVEMPACE) "SOBRE EL FIN DEL HOMBRE"
}

Joaquín LOMBA. Universidad de Zaragoza

\section{INTRODUCCIÓN}

Ofrezco la traducción por primera vez al castellano $y$, además, de forma completa, de la conocida carta del filosofo zaragozano Ibn Bāŷya, Avempace (ca. 1070-1138), fi-l-gāyat al-insāniyya, Sobre el fin del hombre.

Esta carta, compuesta después de El régimen del Solitario (pues lo cita varias veces), fue editada parcialmente (la primera mitad) por Omar Farrukh en su libro Ibn Bajjah and Philosophy in the Muslim West ${ }^{1}$ basándose solamente en el Manuscrito de la Biblioteca Nacional de Berlín, 5060 WE 87, perdido en la segunda guerra mundial pero hallado en Polonia hace pocos años ${ }^{2}$.

La primera edición completa de la carta la hizo Ma'șumi empleando el manuscrito de Oxford, Biblioteca Bodleyana, Pococke 206 (fol. $182 \mathrm{v}-184 \mathrm{r}$ ), y la edición anterior de Farrukh.

${ }^{1}$ Omar Farrukh, Ibn Bajjah and Philosophy in the Muslim West, Beirut, 1945; segunda edición en Beirut, 1952, p. 65-67.

2 Actualmente se encuentra en Cracovia, Biblioteka Jagielonski, Preuss. Staatsbibliothek.

${ }^{3}$ Sagir Hasan Ma'ṣumi, "Ibn Bājjah on Human End", en Journal of Asiatic Society of Pakistan, 2, 1957, p. 182-196. Al texto acompaña una introducción y una traducción al francés, en las páginas 190-196.

SHARQ AL-ANDALUS, 10-11 (1993-1994). HOMENAJE A Ma JESÚS RUBIERA MATA 
M. Fakhry, en 1968 hizo otra edición completa de la carta utilizando sólo el manuscrito dicho de Oxford, Pococke $206^{4}$.

Por fin, Thérèse-Anne Druart publicó una traducción parcial al francés ${ }^{5}$, basándose en todas las ediciones anteriores árabes, consultando, por su parte también, el manuscrito de Oxford, Pococke 206. La traducción es parcial, porque comienza en el folio $183 \mathrm{r} 5$, donde empieza a hablar Avempace sobre los aspectos psicológicos y metafísicos, propiamente tales, como la autora señala. Igualmente indica en su traducción que el manuscrito de Oxford, consultado por ella, carece de signos diacríticos, lo cual, en los casos de duda lo hace señalar en notas a pie de página. Notas, por lo demás, sumamente oportunas y acertadas pues, aunque dice seguir básicamente la edición de Fakhry, sin embargo indica las variantes de las demás ediciones y manuscritos.

En conversación con la Profesora Druart, vimos la oportunidad y necesidad de que se diese a conocer la carta Sobre el fin del hombre en castellano y en su totalidad. Y la oportunidad se basaba en que esta breve carta conecta muy bien con todo el resto del pensamiento de Avempace, aportando detalles de interés para la interpretación de su filosofía.

Para la presente traducción he empleado todos los materiales anteriormente dichos, incluidas las anotaciones y comentarios de Thérèse-Anne Druart. Por mi parte, me he ajustado lo más posible a la literalidad del texto árabe, incluso a costa de la fluidez del castellano. $\mathrm{Y}$ ello, por lo en extremo conciso $\mathrm{y}$ a veces sumamente oscuro de muchos pasajes, como suele ser bastante habitual en Avempace. Por eso, si en algún momento he considerado que las versiones de Druart o de Ma'șumi aportaban algún nuevo aspecto del texto, lo he hecho constar a pie de página para que el lector tuviera una idea más adecuada del pasaje y un espectro más amplio de posibles interpretaciones.

Por lo demás, no voy a hacer aquí un estudio del tema del fin del hombre en Avempace. Lo dejo para otra oportunidad remitiéndome, por el momento, a algunos trabajos que ya se han hecho sobre el tema,

${ }^{4}$ Majid Fakhry, Avempace. Opera metaphysica, Beirut, 1968, p. 98-104.

5 Druart, Th. A., "La fin humaine selon Ibn Bajjah (Avempace)", en Bulletin de Philosophie Médiévale, 23, 1981, p. 59-64. 
como es, por ejemplo, el magnífico estudio de Altmann ${ }^{6}$. Lo que sí es cierto es que el problema le preocupa mucho a Avempace. En efecto, la Carta del adiós ${ }^{7}$ la dedica prácticamente entera a este tema, iniciándola con estas palabras sumamente significativas: después de saludar al destinatario de la carta, su compatriota y amigo Abü l-Ḥasan 'Alī ibn 'Abd al-"Azīz ibn al-Imām, le expone el porqué de este escrito diciendo : "Pero hay una cosa más fundamental y que más necesito decirte, a la vez que es la más agradable y deseable para mi, y es el enseñarte las cosas sublimes que yo sé, a saber, la descripción del fin al cual conduce la naturaleza [del hombre] cuando sigue [aquel camino]. Este [fin] ya lo han descrito largamente los que me han precedido. Uno de los que han descrito y tratado muchas veces es Abü Nașr [al-Fārābī] ${ }^{9}$. $Y$ el lugar [que ocupa] esta ciencia es el suyo propio. Sin embargo, no he encontrado en la totalidad de los libros suyos que han llegado a alAndalus sobre este tema lo que yo he llegado a conocer de este asunto. Se te hará patente, por lo que voy a decir, que este método de especulación únicamente se me ha evidenciado a mí. Ciertamente que he encontrado esto en Aristóteles, en el libro once de la Ética ${ }^{10}$. Pero

${ }^{6}$ Altmann, A., "Ibn Bājja on man's ultimate felicity", en Studies in Religious Philosophy and Mysticism, Londres, 1969, p. 73-107; Kraemer, J. L., "Ibn Bajja y Maimónides: sobre la perfección humana", en Congreso Internacional de las tres culturas, p. 237-245. Para más bibliografía véase mi introducción al pensamiento de Avempace, en Avempace, Diputación General de Aragón, Zaragoza, 1989.

7 "La carta del adiós", trad. de M. Asín Palacios, en Al-Andalus, VIII, 1943, p. 1-87.

${ }^{8}$ Carta del adiós, op. cit., p. 42. Quiero indicar que, en adelante, citaré las obras traducidas por Asín Palacios, con su paginación correspondiente. Sin embargo, en ocasiones modificaré la versión para mejor acomodarla al contexto. De este modo, el lector tendrá a la vista ambas versiones con una mayor riqueza de matices.

${ }^{9}$ Probablemente alude aquí a las obras de al-Fārābī, Kităb tahsil al-sa'ăda (Libro del logro de la felicidad) y Kitab al-tanbih 'ala sabtl al-sa'ada (Libro de la advertencia sobre el camino de la felicidad).

${ }^{10}$ En primer lugar, la Ética de Aristóteles sólo tiene diez libros. Donde trata de este asunto es en el libro VII, donde dice que la felicidad consiste en la vida teorética, que es la única auténticamente divina, frente a la animal y aun política, que son simplemente humanas. 
lo resume muchísimo de tal manera que no es posible que baste con esto solo para saber el asunto en su [verdadero] valor. Pero la causa que pienso existe de que la gente haya dejado de tratar esto no corresponde a lo que vamos tratando [ahora]. Sin embargo, cuando lo conozcas, se te hará evidente, Dios te honre, la gran utilidad que hay [en este camino] pues no hay cosa más útil que este punto para ésta y para la otra vida». No es extraño, por tanto, que dedique una carta, como la presente, a este mismo tema. Por eso, todo su contenido hay que conectarlo, no sólo con la Carta del adiós, sino también con el resto de su obra, en particular, con El régimen de solitario y el Tratado de la unión del Intelecto con el hombre. Es la razón por la que me he permitido citar algunos lugares paralelos, a modo de ejemplo, sugerencia e ilustración del lector. Pero las referencias se podrían multiplicar muchísimo más.

Finalmente, hay que decir que la carta consta de dos partes claramente diferenciadas: en la primera expone los temas del continuo temporal y espacial y del movimiento (lo cual tiene un claro paralelo con lo que dice en la ya citada Carta del adiós, como indico en nota a pie de página) $y$, en la segunda, habla ya directamente del fin del hombre y de los diversos pasos que éste puede dar hasta llegar al fin supremo de la contemplación intelectual y unión con el Intelecto Agente y los Inteligibles.

\section{TRADUCCIÓN}

\section{SOBRE EL FIN DEL HOMBRE.}

Abū Bakr ibn al-Ṣāiig ibn Bāŷŷa.

[Fol. $182 \mathrm{v}]^{11}$ En el nombre de Dios, Clemente y Misericordioso.

${ }^{11}$ Los folios marcados son los del manuscrito de Oxford, reproducido en la edición de Fakhry. 
Las cosas numerables, [pueden] ${ }^{12}$ no tener magnitudes, como las letras y cosas semejantes, y [pueden] estar provistas de magnitudes, como las [distintas] especies de cuerpos. De éstas, hay unas que están en un lugar, otras que no están en el tiempo, como son los inteligibles, y otras que están en el tiempo, como son los movimientos y los seres móviles y, en suma, las cosas generables y corruptibles.

Hablar de lo que está en el lugar, no pertenece a lo que nos proponemos. Pero [en cambio], respecto a lo que está en el tiempo [digo que] ${ }^{13}$ o bien es continuo en sí mismo o lo es respecto al tiempo. Son continuos en sí mismos todos los movimientos; y son continuos en relación al tiempo, por ejemplo, los seres generables y corruptibles.

Todo lo que es continuo tiene partes; ahora bien, lo que es continuo, o es generable o no lo es. Lo que no es generable, o bien se hallan sus partes [todas] a la vez, como es solamente el caso de los cuerpos celestes, o sus partes no se dan simultáneamente y a la vez.

Supongamos un continuo [cuyos extremos son] A B y que tiene [un número] ilimitado de partes, a saber: A, Ŷ, D, H, B, D. Pues bien, cuando se da $\hat{Y}$, es que necesariamente ha habido ya $A$, y cuando ha habido $\mathrm{D}$, forzosamente ha habido $\hat{Y}$ y asi del resto de las partes. $Y$ esto es el movimiento. Pero puede ocurrir que no se dé esto de forma necesaria sino que A y $\hat{Y}$, o más, se den simultáneamente, como es el

${ }^{12}$ Las palabras o frases puestas entre corchetes son añadidos míos para mejor entender el texto.

${ }^{13}$ En to que se va a tratar a continuación sigue Avempace las enseñanzas de Aristóteles en la Física, sobre todo en el libro VI, capítulos 1 y 2, 231 a 20-233 b 33. Del continuo temporal o espacial habla también Avempace en La carta del adiós, op. cit., p. 66, donde dice: «el continuo se dice en primer lugar de las magnitudes, en segundo lugar del movimiento de traslación y, en tercer lugar, del tiempo. Todo lo que está en el tiempo, en cuanto que está en el tiempo, también se dice [que es continuo] de varias maneras: una de ellas, lo que es perpetuo se dice que es continuo, como cuando se afirma del movimiento circular que es continuo. También se predica a veces [la continuidad] de aquello que tiene uno de sus extremos en el tiempo, como cuando se habla de la felicidad de los bienaventurados o de los castigos de los que están en el infierno [y se afirma] que [todo esto] es continuo porque no tiene un extremo final, o como cuando se dice que la no existencia del mundo fue continua hasta que terminó, antes que Dios crease el mundo. Y en general, lo que es perpetuo, en cuanto que es perpetuo, se dice que es continuo». 
caso de los individuos humanos, pues en ocasiones pueden existir a la vez el hombre, su abuelo y los que hay entre ambos. Pero que existan de esta manera [la totalidad de las partes] no es posible, pues, si lo fuera, existiría lo infinito actualmente ${ }^{14}$. Aquello cuyas partes no existen simultáneamente, y es infinito, es lo que es continuo, siendo ese ser uno numéricamente, puesto que sus partes no son separables de los límites que las especifican en la existencia. Y respecto a aquello cuyas partes existen simultáneamente, es de varias clases, a saber: de lo discontinuo y de lo numerado. La primera es la cantidad continuo y la segunda la cantidad discontinuo. Y cada una de sus partes es llamada individuo. Es evidente que las primeras partes en existir de los seres materiales son las que tienen sus partes juntas; y luego el continuo.

Lo discontinuo es el peor de los seres en cuanto a existencia, y tiene varias especies, la más baja de las cuales en cuanto a existencia es la menos [separable], y la más noble la del animal [Fol. $183 \mathrm{r}$ ] que se reproduce, en el cual no hay debilidad ${ }^{15}$. Así, por ejemplo, el animal que engendra un animal en sí mismo. Éste también tiene varios niveles. Uno de ellos, es [el de] los elementos cuyas partes todas no existen simultáneamente, ni existe una parte tras otra, sino que existe simultáneamente y siempre un conjunto de las mismas perdiéndose unas partes y delimitándose otras. Pero la explicación de esto se halla en otro lugar. Es evidente que aquello cuyas partes existen una tras otra, es similar en este sistema a lo eterno y que la multiplicación hace las veces de la perpetuidad $^{16}$.

${ }^{14}$ En el original dice: ma ${ }^{\text {tan }}$ es decir: a la vez. Pero parece que el sentido es el que le doy arriba: actualmente, simultáneamente.

${ }^{15} \mathrm{Ma}$ 'sumi lee: fatra, que significa languidez, torpor, debilidad. El traduce por "interregnum". En castellano resulta más expresivo: "debilidad" o "torpor". Pero Fakhry lee: katra, multitud, abundancia. Creo más oportuna la lectura de Ma'sumi.

${ }^{16}$ Usa aquí deliberadamente dos términos distintos, a saber: abadiyy ${ }^{\text {un }}$, que traduzco por eterno, y dawäm, que traduzco por perpetuo, permanente. Parece que la distinción es deliberada: lo eterno supone una categoría extratemporal que no está sujeta a la duración sucesiva del tiempo, la cual, cuando es infinita, resulta ser la perpetuidad. 
Las potencias activas ${ }^{17}$ y las pasivas son la causa de la existencia y es por ellas por lo que algo existe. Veamos las potencias activas humanas, pues las pasivas pueden ser materiales o animales y el hombre es demasiado excelso como para que se le relacione con ellas. La facultad de aprender es una potencia pasiva, pero bajo otro aspecto. En suma, no es el propósito de este discurso resumir todas ellas, sino [solamente] aquello que conviene comprender.

La potencia activa es como el arte de la gramática, por la cual se hace la gramática ${ }^{18}$. Ella persigue únicamente la perfección, deteniéndose [luego]. La repetición de su acto, únicamente se lleva a cabo con el alma apetitiva y con la opinión. Ahora bien, lo que es por causa del alma apetitiva, es como lo que hace el agente que únicamente desea hacer. Y lo que es por opinión consiste en actuar para conseguir otro objetivo, cual es la utilidad de aquel acto. Es evidente que el alma apetitiva desea algo perpetuo $\mathrm{o}^{19}$ algo en cuanto que es perpetuo. A este deseo se le llama actividad y la privación de este deseo es pereza, apatía y otras cosas parecidas. Con este deseo se lleva a cabo el acto que produce la perpetuidad a partir de las potencias. Y este deseo es puramente animal no siendo en absoluto privativo del hombre. Por eso, todo el que hace un acto cualquiera de esta manera, realiza un acto animal. Es evidente que el hombre, cuando actúa de esta forma, únicamente actúa no en cuanto que es hombre sino en cuanto que es animal [dotado] de imaginación humana. Y es claro que de la eternidad únicamente consigue esta cantidad, a saber, sólo la cantidad de perpetuidad que se ha concedido a las facultades animales ${ }^{20}$.

El que actúa por opinión, únicamente actúa en cuanto que es hombre. Esta opinión o mueve hacia lo que es perpetuo por su esencia

17 Aquí comienza la traducción de Th.A. Druart citada arriba.

${ }^{18}$ En este pasaje, sigo, como lo hace Druart, la lectura de Fakhry: nahw, gramática. Farrukh y Ma'șumi leen: taŷr, comerciante. Druart indica que, en primer lugar, el Ms. no da ningún signo diacrítico y la grafía de wāw no difiere de la de rā, en la palabra nahw; y, en segundo lugar, que no se sabe si el Ms. de Berlín da pie a interpretar taŷr en lugar de nahw.

${ }^{19}$ Farrukh lee: aw, o, en lugar de wa, y, que es lo que propone Fakhry. Prefiero la lectura primera.

${ }^{20}$ Farrukh lee: lahu al-quwa; Fakhry, li-1-quwa. Sigo la versión de Fakhry. 
o hacia lo que es perpetuo por multiplicación. Si se trata de lo perpetuo por multiplicación, aquel fin toma el lugar de la preparación, siendo aquel fin equívoco, bien sea porque [se busca con] el deseo, y entonces es animal, bien sea porque [se busca con] la opinión, y entonces no transciende a lo infinito. Habrá, por tanto, un fin que se busca por sí mismo y que, cuando se consigue, es suficiente ${ }^{21}$.

Estos fines difieren según $\operatorname{sean}^{22}$ las naturalezas de los individuos humanos. En efecto, entre la gente, hay quien se prepara sólo para la zapatería, y quien se dispone para otras cosas. Pero los fines, según se demostró en muchos lugares, sirven unos a otros, acabando todos en un único fin. En efecto, el fin humano es uno solo, el cual es el principal $^{23}$, estando cada uno de los otros fines, sean cuales sean, subordinados a éste. El hombre señor por naturaleza es el que se prepara para este fin. Y quien no se prepara para este fin, está por naturaleza gobernado. Por eso hay gentes que están por naturaleza gobernadas y las hay que tienen el señorío por naturaleza. Unos dominan a los demás $y$ otros son dominados por otros ${ }^{24}$. Se demostró en muchos lugares que aquel fin es eterno, que no es generable ni corruptible y que es o los inteligibles o uno de ellos ${ }^{25}$.

${ }^{21}$ La versión de Ma'ṣumi es: "This end is equivocal either due to propensity only -in which case it is animal- or due to opinion which is not unlimited, and is therefore intended only due to itsself which is, whenever achieved, regarded sufficient".

${ }^{22}$ Fakhry lee: bi-sabab, por causa de; Farrukh: bi-ḥasab, según.

${ }^{23}$ Farrukh: al-ra'isiyya; Fakhry: al-ra'isa.

${ }^{24}$ Qawm, en lugar de gente puede traducirse por pueblos: "Unos pueblos dominan a los demás y otros pueblos son dominados por otros". Esta idea del autodominio, de la autarquía natural, parece que es algo que tiene Avempace en el centro de su pensamiento. Él la cifra en la vida especulativa, en el desarrollo máximo del intelecto y la contemplación de los inteligibles, que es el fin supremo del hombre. En la Carta del adiós, p. 65, dice: "Observamos, en efecto, que hay quien ha nacido con talento natural para ser carpintero y que no vale para otra cosa. Ese tal será apto tan sólo para servir a otro [...]. En cambio, el hombre dotado de perfecto talento natural será aquel que haya nacido dispuesto naturalmente a ser algo para sí mismo. Es evidente, por tanto, que el talento superior a todos será aquél con que se adquiere la ciencia especulativa".

${ }^{25}$ Todo este pasa je encuentra su paralelo en la Exposición a la República de 
Dejemos $^{26}$ de explicar ahora de qué inteligibles se trata para que no se alargue el discurso ${ }^{27}$. [Fol. $183 \mathrm{v}$ ] Cuando se da un acto [dirigido] hacia este fin, surge de la opinion ${ }^{28}$ y de algo eterno. $Y$, de este modo, se da también la eternidad en el sujeto que actúa [así] en la medida en que se aproxima a ella. Bajo este aspecto, aquel acto es eterno por su esencia, porque aquello en que se basa es eterno, ya sea próxima o remotamente. El alma impulsiva, puesto que está privada de esta especie de perpetuidad, ansía lo que se le asemeja y repite el acto. La opinión y el alma apetitiva desean ambas la perpetuidad, sólo que la opinión la desea por si misma y el apetito desea lo que se le parece. La opinión no es recta por sí misma pero se hace perfecta o se da cuenta

Platón, de Averroes (trad. de Cruz Hernández, Madrid, 1986, pp. 93). En Averroes, se está postulando que el saber teórico es el fin último y único del hombre, al cual está subordinado el saber práctico y todas las otras artes que dependen de las superiores, del saber teórico y del fin último como los efectos de sus causas. Tal vez quede aclarado lo conciso del texto de Avempace, por el lugar que creo más o menos paralelo de Averroes: "Respecto de la parte teórica ya aparecía en los libros físicos que la naturaleza del hombre no está fundada en la necesidad, sino en la perfectividad, ya que lo que existe en razón de ser mejor debe preferirse sólo por necesidad. Por tanto, esta parte de la razón, es decir, de la práctica, está fundada necesariamente en la teórica, lo que coincide con lo que sabemos de su carácter. Es evidente, pues, que dichas artes existen originariamente en razón de sus acciones, como éstas en función de la justicia. Ahora en virtud de la adquisición de las artes un hombre deberá ser gobernado y vasallo, y otro deberá ser señor. El señor lo será en virtud de una disposición por la cual está más capacitado para el gobierno: y este hábito no es nada diferente de la parte de la razón llamada teórica. El modo como esta parte de la razón enseñorea sobre la otra, es decir, sobre la práctica, en el alma individual, es el mismo como el de quien posee las disposiciones para adquirir las ciencias teóricas para gobernar a aquellos cuyos hábitos naturales sólo son adecuados para las artes prácticas. Luego éstos últimos son siervos por naturaleza y deben ser naturalmente gobernados. Esta relación de cada una de estas dos partes del alma es necesariamente una correlación, es decir: la relación del gobernante y del gobernado" (ibidem., p. 86).

${ }^{26}$ Sigo a Farrukh que lee: fa-li-natruk. El Ms. y Fakhry, en cambio, dicen: fa-li-nanzil. Concuerda mejor la versión que adopto a la vista de la variación y nota que sigue.

${ }^{27}$ Fakhry y el Ms. omiten la secuencia li 'ari yatüla al-qawl que inserta Farrukh.

${ }^{28}$ Aquí termina la edición de Farrukh. 
de este fin que es perpetuo ${ }^{29}$, pues el ser ansía la perpetuidad. El alma apetitiva únicamente desea la forma imaginativa intermedia ${ }^{30}$, sobre la cual se habló en el libro de [El régimen del Solitario] $]^{31}$, y la forma imaginativa $^{32}$. Aquellas dos formas no son perpetuas y el alma apetitiva las desea. Pero esas formas son muchas. Por eso vacila el alma apetitiva en el esfuerzo de ir tras ellas: unas veces aparece ésta y otras aquélla, cuando las examina. Además, el alma apetitiva se sirve de la naturaleza. Por eso le alcanza el aburrimiento y el tedio cuando no le favorece la naturaleza, pues la naturaleza no es simple, razón por la cual no permanece en un solo y mismo estado. Por causa de la naturaleza, necesita el animal de reposo. Y por causa del alma apetitiva se queja el hombre de que el reposo perdure. Estas dos formas ${ }^{33}$ son corruptibles y no son eternas. Por eso el alma apetitiva no es consciente de la perpetuidad sino de lo que se le parece ${ }^{34}$.

${ }^{29}$ Druart traduce así: "L'opinion n'est pas bonne en soi. Elle est parfaite (?) ou cherche à connaître cette fin qui est perpétuelle".

${ }^{30}$ Intermedia porque está entre la forma espiritual universal y la sensible singular material.

${ }^{31}$ Ver Avempace, E1 régimen del solitario, trad. de Asín Palacios, passim y, sobre todo, en p. 49-52 y, más en particular 74-76, donde comienza hablado de "la clase segunda de actos con los que se intenta conseguir formas espirituales, son aquéllos que tienden a la forma espiritual que reside en la imaginación".

${ }^{32}$ A saber, la que es totalmente particular. Sobre la imaginación y las formas imaginativas, puede verse el comentario al De anima, capítulo X. Ver Ibn Bajjah's. 'Ilm al-nafs, traducción al inglés y notas de Ma'ṣumi, Karachi, 1961, p. 187-192.

33 A saber: la forma imaginativa intermedia y la forma imaginativa concreta, a las que se ha aludido más arriba.

${ }^{34}$ Es interesante recordar lo que dice en El régimen del solitario, op. cit., p. 95: "Los actos intelectuales y las ciencias, son perfecciones en sentido absoluto y sin restricción, siendo característicasdel hombre y no compartiéndolas con otros seres distintos al humano. Estos [conocimientos] o dan la existencia perpetua o bien son medios para lograrla. En cambio, las formas espirituales particulares, solamente proporcionan la existencia famosa, y las formas materiales sólo dan la existencia que se refiere a ellas mismas [es decir, la material] que es la más breve de todas, pues la existencia material es la más apropiada para desaparecer, mientras que la existencia intelectual es la más digna de perpetuarse. Las [formas] espirituales son las de más larga permanencia, en comparación con las inteligi- 
La opinión recta por su esencia, no por accidente, desea lo perpetuo por sí mismo. Pero la opinión a veces no es recta en sí misma, y sin embargo lo es, por accidente, para quien la profesa. Por ejemplo, las opiniones de los astutos y de los embusteros. En efecto: la opinión de éstos es recta de acuerdo con los propósitos que se fijan, pero no lo es por sí misma, porque el fin perseguido con ella es falso e inicuo. Estas [opiniones] $^{35}$ a veces son rectas relativamente pero no de forma absoluta. Tal es el caso de la coloquíntida pues ella es útil para los flemáticos, no siendo útil de forma absoluta. En cambio, el pan y la carne son útiles de forma absoluta y según la naturaleza. Del mismo modo, a veces, la opinión recta de forma relativa puede ser recta de modo absoluto, siendo entonces enteramente buena. Puede ser también [la opinión] relativamente recta sin que lo sea de modo absoluto, siendo entonces buena bajo un aspecto y mala bajo otro. El hombre, naturalmente, sólo conoce la opinión relativamente recta, pero no conoce la rectitud absoluta, salvo los sabios después de haberse dedicado a la perfección del hombre. Esto ya se explicó en la ciencia política ${ }^{36}$. Esto es todo lo que tengo que decir de este asunto según el método científico.

Decimos: ¿Acaso, oh hermano piadoso, cuando reflexionas sobre lo que te he dicho antes y lo relacionas con el resto de las cosas que

bles. Y esto es verdad porque están compuestas de los dos extremos: del material y del inteligible. De lo inteligible se sacan una existencia larga y una permanencia durable. En cambio de lo material, se obtiene una permanencia finita, pues toda su duración es limitada y su largura es breve en comparación con la eternidad, ya que no hay comparación alguna con ella; la largura de la duración finita es limitada. Sin embargo, si el hombre ama por naturaleza el durar un año más en su vida, con mayor razón deseará añadir [a su existencia] más y más decenas, centenas, milenios y aun más todavía, como vemos en muchos profesionales y en muchos científicos [que desean vivir] cientos y aun miles [de años], por ejemplo Alejandro, de entre los reyes, y otros muchos sabios [que quieren vivir] millares de años, como Hipócrates y otros similares".

${ }^{35}$ Druart interpreta "estos [fines]" en lugar de "estas [opiniones]".

${ }^{36}$ Suele aludir Avempace en sus obras a un cierto tratado de Política. La lista que nos da Ibn al-Imām alude a unos Captulos de politica, sobre las diversas formas de gobierno y sobre el método de vivir que debe seguir el solitario. Pero no se tiene manuscrito alguno, al menos de momento. Tal vez pueda referirse también a El régimen del Solitario. 
sabes $^{37}$, y reflexionas todas las cosas, no te es evidente que el ser se predica de manera equívoca, no unívocamente, con respecto a los dos estados del hombre? ${ }^{38}$. Es decir que la existencia del hombre como regidor $^{39}$ que es, de forma bien sabida, de las cosas que conocemos del mundo en nuestro tiempo, solamente [se ve movida] por el alma apetitiva. De esta manera hay cosas que son rectas por si mismas, como es el uso de alimento, de las ropas ${ }^{40}$ y de cosas que no te es difícil enumerar si recuerdas lo que hay en el libro de [El régimen del] Solitario $^{41}$. Ni una sola de estas cosas es considerada noble o excelsa [Fol. $184 \mathrm{r}$ ], ni creemos que [constituyan] una perfección en alguno de los modos de vida conocidos. Por el contrario, éstas son consideradas dentro de las opiniones rectas ${ }^{42}$ en la medida en que van más allá de aquellas en las cuales el alma apetitiva busca la forma estimativa, bien sea la intermedia bien sea la imaginativa. Ya se demostró en el libro de [El régimen del] Solitario que algo perteneciente al género de ambas formas se da a veces en el animal irracional y que, por consiguiente,

${ }^{37}$ Fakhry transcribe: ta lamu-hu, que es la lectura que he seguido, "que sabes". Ma'ṣumi y el Ms., en cambio: ta'mulu-hu: "que esperas".

${ }^{38}$ Se entiende: de la rectitud y del pecado o error.

${ }^{39}$ Emplea la palabra mutadabbir, de la misma raíz y forma verbal que el tadbīr de El régimen del Solitario, al que hará mención un poco más abajo.

${ }^{40}$ Fakhry lee: ditār, ropa, capa. Ma'ṣumi, en cambio: diyār, casas. El Ms. no pone puntos diacríticos.

${ }^{41}$ El régimen del solitario, op. cit., por ejemplo, entre otros pasajes, el siguiente: p. 75 y ss. donde, por ejemplo, dice Avempace: "La segunda clase de actos que tiende a las formas espirituales que están en la imaginación, son de varias clases: una de ellas es la que intenta [producir] cierta especie de impacto [en los demás] como es el vestir armas fuera del tiempo de guerra, o tener un aspecto serio $u$ otras formas externas [reflejo] de lo anímico. Aquí entran también Ios artificios que usan los reyes de servirse de aquellos utensilios cuyo aspecto inspira terror, cuando van a verles las gentes, los extranjeros o los embajadores, como es el vestirse las armas en las asambleas públicas y otras cosas que se encuentran escritas en las historias de los reyes de las naciones."

${ }^{42} \mathrm{Tal}$ vez sea mejor la lectura de Ma'șumi: al-șāiba, rectas, en lugar de la de Fakhry: al-lisāniyya, que podría significar algo referente a los bandidos, ladrones, bandoleros. El Ms, ofrece una lectura oscura. 
[estas formas] son animales ${ }^{43}$. Las opiniones relativamente rectas están en esta especie de actos y pueden ser rectas, a veces, de modo absoluto. Y en esta especie entra el aprender y el descubrir. Éstas, puede que no sean rectas ni absolutamente ni relativamente, sino que se trata o bien de un juego y distracción, o de una fatiga y futilidad. A veces [pueden constituir también] una alegría.

La más elevada de las categorías de modo de vida bien conocido es aquélla cuyo poseedor es capaz de ejecutar aquello a lo que le conduce el alma apetitiva; [a saber, cuando el alma apetitiva] busca la forma estimativa antes de que ejecute su sentencia de cólera contra cualquier individuo que le salga al encuentro, y que logre cualquier ${ }^{44}$ individuo que desee, bien sea en tanto individuo por si mismo, siendo entonces movido por la forma imaginativa, bien sea en tanto que individuo de la especie, siendo en tal caso movido por la forma intermedia. Lo que se parece a esto no es difícil valorar. Pues la excelsitud y perfección en estos tales consiste en el dominio del alma apetitiva y en su liberación de sus ataduras. Por eso, aquél a quien alcanza este nivel muere como los reyes antiguos ${ }^{45}$, los cuales dominaron la mayor parte del mundo habitado, [partiendo] de sus primitivas situaciones ${ }^{46}$, y no sacaron

${ }^{43}$ Ver El régimen del Solitario, por ejemplo, entre otros muchos pasajes, p. 91-94. Particularmente, puede referirse a lo que dice en la página 93, en donde dice: "En cuanto a la forma espiritual particular, se dan en muchos animales algunas acciones que se obtienen con ella, como es la vergüenza en el león, la vanidad en el pavo real, el hacer caricias en el perro, la nobleza en el gallo, la astucia en el zorro. Pero estas cualidades, cuando se trata de animales, son propias naturalmente de la especie, no perteneciendo de modo particular a los individuos de esta especie, sino que las adquiere el sujeto individual, por medio de formas espirituales particulares, en cuanto que es individuo de esa especie, la cual proporciona esa cualidad o estado, ennobleciéndole así dicha especie; no son propias del individuo. Esto sólo se da en el individuo humano. Las especies en las que se encuentran estas cualidades excelentes, son las más nobles de entre los animales. $Y$ si estas cualidades no son virtudes, es porque [el animal] las emplea en todo momento, convenga o no convenga [llevarlas a cabo]. Por eso no son virtudes, sino por equivocidad".

44 Se opta la versión de Fakhry: ayy, en lugar de la del Ms. y Ma'ṣumi: 'ilā.

${ }^{45}$ Prefiero la lectura de Fakhry: al-gäbirīn antiguos, en lugar de la de Ma'ṣumi: al-q̨āhirīn, victoriosos, vencedores.

${ }^{46}$ La frase es de dudosa interpretación: 'alà mṭl hālihim al-'ūlà. Druart 
ningún provecho de sus acciones ni de sus vidas ni de sus amplios poderes victoriosos y gran fortuna. Por eso muere la mayor parte de ellos en estado de angustia por aquello a lo que llegan y con tristeza por la pérdida de lo que han tenido. Les sobreviene el trabajo y la fatiga al afanarse el alma apetitiva con el cansancio de los instrumentos naturales. El recuerdo de aquellas ilusiones pasadas queda en sus almas y su idea les hace llorar lamentándose de ello. Y si ocurre esto en tales reyes, ¿cuánto más en los que son inferiores a ellos? Realmente esto es así porque el afán de esta alma es reunir lo que no se reúne y lograr lo que no se logra, pues el asunto no tiene límites. Por eso no alcanza el animal irracional este tipo de sufrimientos, porque no recuerdan semejantes ilusiones. El alma apetitiva en [el animal irracional] no las desea, y los únicos sufrimientos que le alcanzan son los que le vienen de la naturaleza, a saber: del envejecimiento, lo cual acontece a todo cuerpo natural.

El hombre que se ocupa de la opinión recta de modo absoluto, tan sólo desea su alma apetitiva o bien la [forma] intermedia en cuanto que está conectada con la cosa eterna, o deseando la cosa eterna por sí misma. Ahora bien, si desea la forma intermedia, como a su fin, solamente se le da la cosa eterna en esa ${ }^{47}$ medida. Pero si considera la cosa eterna, se esfuerza por ella y no repite un acto deseando lograr lo que se parece a lo perpetuo, sino que lo repite para conseguir lo eterno, no conviene que se le llame a aquello repetición en sentido ordinario. En efecto, cuando el carpintero repite el golpe con el hacha, no se trata de una repetición sino de una parte del trabajo. Esto es evidente. Lo que le sobreviene es únicamente la cosa permanente. Por eso, este hombre, cuando actúa así, únicamente actúa por el alma apetitiva, o bien en las cosas necesarias para lograr la situación en que ${ }^{48}$ se basa $^{49}$ la conse-

traduce: "selon la ressemblance de leur condition première". Ma'sumi: "which [the world] were in their elementary state". Creo que lo que quiere decir, tal vez, es que tales reyes pasaron de una situación simple de pobreza originaria a dominar todo el mundo habitado, lo cual no les dejó en absoluto satisfechos.

${ }^{47} \mathrm{Ma}$ 'ṣumi lee: bi-dālika. Fakhry: dālika.

${ }^{48}$ Es mejor fihā, tal como lo lee Ma'ṣumi, en lugar de qad que lee Fakhry.

49 Tal vez mejor ya'tamidu, tal como lo propone Ma'șumi, en lugar del ta'tamidu de Fakhry. Pococke no pone puntos diacríticos. 
cución del fin, o bien en las cosas bajas ${ }^{50}$, para que vea aquella clase de inteligibles. Asf, participa en algunas de sus acciones del hombre que actúa por el alma bestial, en la manera en que participa el hombre piadoso con el hipócrita. En efecto, ambos se alzan, se humillan y recitan [las oraciones]. Sin embargo, la acción del piadoso es oración y la del hipócrita no es oración, en absoluto, sino algo parecido. Por eso se llama a la acción de ambos oración de manera equívoca. Y cuando alcanza al hombre esta perfección, se libra de la guerra con la naturaleza y de sus sufrimientos, del alma y de sus potencias. Y habrá conseguido, como dijo Aristóteles, la paz en una alegría y contento perpetuos $^{51}$. Pues lo perpetuo, desde el punto de vista del tiempo, no es perpetuo, ya que lo perpetuo es lo que no es contenido en el tiempo, pues es perpetuo por sí mismo, no porque su tiempo sea perpetuo. Por eso, la cosa perpetua más propiamente perpetua es Dios Altísimo, que es quien da la perpetuidad. Por tanto es preciso que reflexionemos sobre la perpetuidad, que examinemos de cuántas maneras se predica y que se explique cómo unirse a lo perpetuo y que se enumeren sus modos ${ }^{52}$.

${ }^{50}$ Druart y Ma'șumi traducen mustakina por "cachées" y por "hidden" respectivamente. Prefiero "bajas", "humildes" porque pienso puede tener el sentido de que el uso del alma apetitiva en el hombre sabio se dirige sólo a lo bajo para remontarse a los inteligibles de que habla: ma'qūlāt.

${ }^{51}$ Puede tratarse de una alusión a la Ética a Nicómaco, X, c. 6 y, sobre todo, el 7 (1177 b 26-1178 a 2), donde se lee, por ejemplo: "La felicidad perfecta consiste igualmente en el ocio. No nos privamos de los ocios más que para conseguirlos y es para vivir en paz para lo que hacemos la guerra [...]. Todos los bienes que corresponden al hombre feliz parecen ser un resultado de esta actividad. Ella constituirá realmente la felicidad perfecta si se prolonga durante toda la vida. Pues nada puede ser imperfecto en las condiciones de la felicidad".

${ }^{52}$ Esta apoteosis intelectual con la que Avempace se desliga de los cambios materiales, de la temporalidad y espacialidad, viene expresada en otros muchos lugares de su obra, como es por ejemplo en la Carta del adiós, p. 83, en la cual distingue entre alma racional (nafs nātiqa) e intelecto ("aql), estribando la diferencia en que el alma racional supone un primer grado de lejanía de lo material y múltiple, siendo, en cambio, lo intelectual, lo absolutamente uno, pleno dador de felicidad total al hombre: "El alma racional, en cambio, puesto que está lejos de la materia, permanece en un mismo estado, sin que tenga contrarios, salvo el hecho de que es múltiple [numéricamente]. Por lo que respecta al intelecto adquirido, como resulta que es uno bajo todos los aspectos y que está en la máxima lejanía de la materia, no le afecta la contrariedad, como le afecta a la 
Termina el discurso sobre este asunto y alabado sea a Dios, a quien se debe la alabanza.

naturaleza, ni tampoco las acciones que son efecto de la contrariedad, como le acontece al alma bestial, ni aun la huella de la contrariedad, como ocurre en el alma racional, la cual entiende los inteligibles materiales que son múltiples. Él es, por tanto, siempre uno y está de la misma manera en el puro placer, en la alegría, en la belleza y en el gozo. Él es el que determina todas las cosas y Dios está satisfecho de él de la manera más perfectamente complacida posible". 